\title{
Revista Colombiana de

\section{Tormenta eléctrica y torsade de pointes asociados \\ a tratamiento con antimoniales en un paciente con leishmaniasis cutánea}

\author{
Laura Arcos ${ }^{\mathrm{a}}$, Carlos Rincón ${ }^{\mathrm{b}}$, Diego Vanegas ${ }^{\mathrm{b}}$ y Ramón Medina ${ }^{\mathrm{c}, *}$ \\ a Cardiología, Hospital Militar Central, Bogotá, Colombia

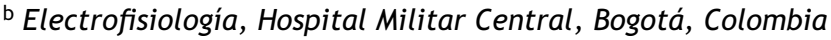 \\ c Medicina Interna, Hospital Militar Central, Bogotá, Colombia
}

Recibido el 7 de diciembre de 2016; aceptado el 22 de septiembre de 2017

Disponible en Internet el 14 de marzo de 2018

\section{PALABRAS CLAVE}

Arritmias;

Síndrome de QT

largo;

Torsade de pointes

\begin{abstract}
Resumen La toxicidad por antimoniales puede comprometer múltiples sistemas y llega a ser potencialmente fatal. Se presenta el caso de un joven militar quien durante el tratamiento de su segundo episodio de leishmaniasis cutánea presentó toxicidad pancreática y cardiaca, desarrollando un síndrome de QT largo adquirido y tormenta eléctrica con episodios frecuentes de taquicardia ventricular que incluían torsade de pointes. Se realizó tratamiento en unidad de cuidados intensivos, donde requirió múltiples descargas de desfibrilador y la administración de sulfato de magnesio hasta la resolución del cuadro clínico.

( $) 2018$ Sociedad Colombiana de Cardiología y Cirugía Cardiovascular. Publicado por Elsevier España, S.L.U. Este es un artículo Open Access bajo la licencia CC BY-NC-ND (http:// creativecommons.org/licenses/by-nc-nd/4.0/).
\end{abstract}

Electrical storm and torsade de pointes associated with antimonial treatment in a patient with cutaneous leishmaniasis

\begin{abstract}
The toxicity due to antimonial can compromise multiple systems, and is potentially fatal. The case is presented of a young soldier, who had pancreatic and cardiac toxicity during the treatment of his second episode of cutaneous leishmaniasis. This developed into an acquired long QT syndrome and an electrical storm with frequent episodes of ventricular that included torsade de pointes. Treatment was given in the Intensive Care Unit, where he required multiple defibrillator discharges and the administering of magnesium sulphate until the clinical symptoms were resolved.

( $) 2018$ Sociedad Colombiana de Cardiología y Cirugía Cardiovascular. Published by Elsevier España, S.L.U. This is an open access article under the CC BY-NC-ND license (http:// creativecommons.org/licenses/by-nc-nd/4.0/).
\end{abstract}

\footnotetext{
* Autor para correspondencia.

Correo electrónico: ramon8809@hotmail.com (R. Medina).
} 


\section{Introducción}

El síndrome de QT largo (SQTL) adquirido o secundario, con frecuencia es un efecto asociado a toxicidad por medicamentos y es una condición clínica capaz de generar arritmias fatales.

El tratamiento con antimoniales constituye en la actualidad la terapia de primera línea en pacientes con leishmaniasis cutánea; sin embargo, las toxicidades potenciales asociadas a esta terapia pueden llegar a comprometer diferentes sistemas y la vida del paciente. Se expone el caso de un paciente con leishmaniasis cutánea quien desarrolló toxicidad cardiaca por antimoniales manifestándose por un SQTL adquirido, y desarrollo de tormenta eléctrica con múltiples episodios de taquicardia ventricular (TV) y torsade de pointes.

\section{Caso}

Militar de 29 años de edad, quien consultó al servicio de urgencias por tres días de dolor en hemiabdomen superior, náuseas, emesis, hiporexia y fiebre de $38,2{ }^{\circ} \mathrm{C}$. Antecedente de leishmaniasis cutánea, el primer episodio hace 6 años, en la actualidad con nueva infección en tratamiento de 15 días con antimoniato de meglumina intramuscular. Al examen físico de ingreso fue positiva la presencia de dolor a la palpación en abdomen superior sin signos de irritación peritoneal y una úlcera, de bordes definidos, de $1 \mathrm{~cm}$ de diámetro en glúteo izquierdo. Los paraclínicos iniciales evidenciaron leucocitosis con neutrofilia leve, elevación de transaminasas y amilasa sérica. Permaneció en salas de observación de urgencias para hidratación endovenosa y fue valorado por el servicio de cirugía general, que descartó abdomen agudo susceptible de tratamiento quirúrgico.

Durante el segundo día de observación presentó dolor torácico tipo punzada de segundos de duración; se realizó electrocardiograma (ECG) que mostró taquicardia sinusal, prolongación del intervalo QTc e inversión de la onda T en derivaciones precordiales (fig. 1); por esta razón fue trasladado a salas de reanimación para monitorización hemodinámica. Minutos después desarrolló paro cardiorrespiratorio de 14 minutos de duración en ritmo de taquicardia ventricular; se realizaron maniobras avanzadas de reanimación, administración de adrenalina, amiodarona y sulfato de magnesio, luego de lo cual retornó a ritmo sinusal. Fue trasladado posteriormente a la unidad de cuidados intensivos con soporte vasoactivo e infusión de potasio endovenoso; con diagnóstico de pancreatitis aguda medicamentosa y síndrome QT largo secundario a toxicidad por antimoniato de meglumina e hipocalemia.

Al tercer día de estancia presentó multiples episodios de taquicardia ventricular monomórfica y torsade de pointes que requieron manejo con desfibrilación y la administración de sulfato de magnesio (fig. 2). Fue valorado por Electrofisiología cardiaca por diagnóstico de tormenta eléctrica, quienes indicaron sedación profunda para disminuir descarga simpática. Se suspendió la administración de amiodarona, se inició isoproterenol endovenoso y se ingresó a sala de fluoroscopia para inserción de marcapaso transitorio con el fin de brindar sobreestimulación ventricular (fig. 3).
Durante el cuarto día del ingreso persistieron los episodios de TV colapsante, hasta entonces diez episodios; evolucionó a disfunción multiorgánica con compromiso cardiovascular, pulmonar, hepático y renal. Presentó fiebre y se documentó en hemocultivos crecimiento de cocos gram positivos, por lo cual requirió terapia antibiótica. Se mantuvo reposición de potasio con la meta de concentraciones séricas entre 4-5 $\mathrm{mEq} / \mathrm{L}$ y sulfato de magnesio para lograr concentraciones entre 2,5 y $3,5 \mathrm{mEq} / \mathrm{L}$. Se hizo ecocardiograma transtorácico que reportó cardiopatía dilatada incipiente de etiología tóxica con aturdimiento miocárdico por tormenta eléctrica; compromiso severo de la fracción de eyección del ventrículo izquierdo (FEVI 35\%), disfunción diastólica tipo II, insuficiencia mitral mínima e insuficiencia tricuspídea leve.

Posterior al séptimo dia de estancia no presentó nuevos episodios de TV y el ECG evidenció ritmo intrínseco con frecuencia cardiaca entre 80 y 90 latidos por minuto; se inició betabloqueador titulado según tolerancia y se retiró sistema de marcapaso transitorio. Hubo resolución progresiva de signos de falla multiorgánica, el examen neurológico fue satisfactorio y toleró el retiro del soporte ventilatorio. Sin embargo, durante al décimo día posterior al ingreso, en monitor electrocardiográfico, se documentaron múltiples episodios de bigeminismo, con intervalo QTC marcadamente prolongado $(690 \mathrm{~ms})$; se decidió, por tanto, reinicio de isoproterenol y se pasó nuevo marcapaso transitorio.

Posteriormente tras mejoría clínica, fue trasladado a pisos de Cardiología; se sospechó SQTL de penetrancia incompleta desencadenado por antimoniato de meglumina e hipocalemia, por lo cual se solicitó estudio genético y se implantó cardiodesfibrilador como prevención secundaria de muerte súbita y se dio egreso. El ECG previo al alta médica mostró normalización del intervalo QTc (fig. 4).

\section{Discusión}

El SQTL congénito es una canalopatía arritmogénica que afecta la repolarización ventricular debido a mutaciones genéticas que generan susceptibilidad al desarrollo de muerte súbita por arritmias ventriculares malignas tipo torsade de pointes. Fue descrito por primera vez por Anton Jervell y Fred Lange Nielsen en 1957 (mutaciones homocigotas asociadas a sordera congénita) y posteriormente por Romano y Ward en 1964 (mutaciones heterocigotas sin sordera neuronal). A la fecha se han descrito alrededor de 13 síndromes; entre ellos los más frecuentes son los SQTL tipo 1 y 3. La incidencia aproximada es de 1 caso por cada 2.500 nacidos vivos; el $32 \%$ de los portadores son asintomáticos y pueden tener QTc normal ${ }^{1-3}$.

Los canales iónicos presentes en el miocardio son proteínas transmembrana que forman complejos macromoleculares compuestos por una subunidad formadora del poro del canal (alfa) y una subunidad auxiliar (beta). Las mutaciones de los genes que codifican la subunidad alfa constituyen los tres subtipos más frecuentes, que corresponden al $65 \%$ de los SQTL congénitos: el SQTL tipo 1 (canales lentos de potasio $\mathrm{IK}_{\mathrm{s}}$ ), el SQTL tipo 2 (canales rápidos de potasio $\mathrm{IK}_{\mathrm{r}}$ ) y el SQTL tipo 3 (canales de sodio Nav 1.5$)^{1}$. 


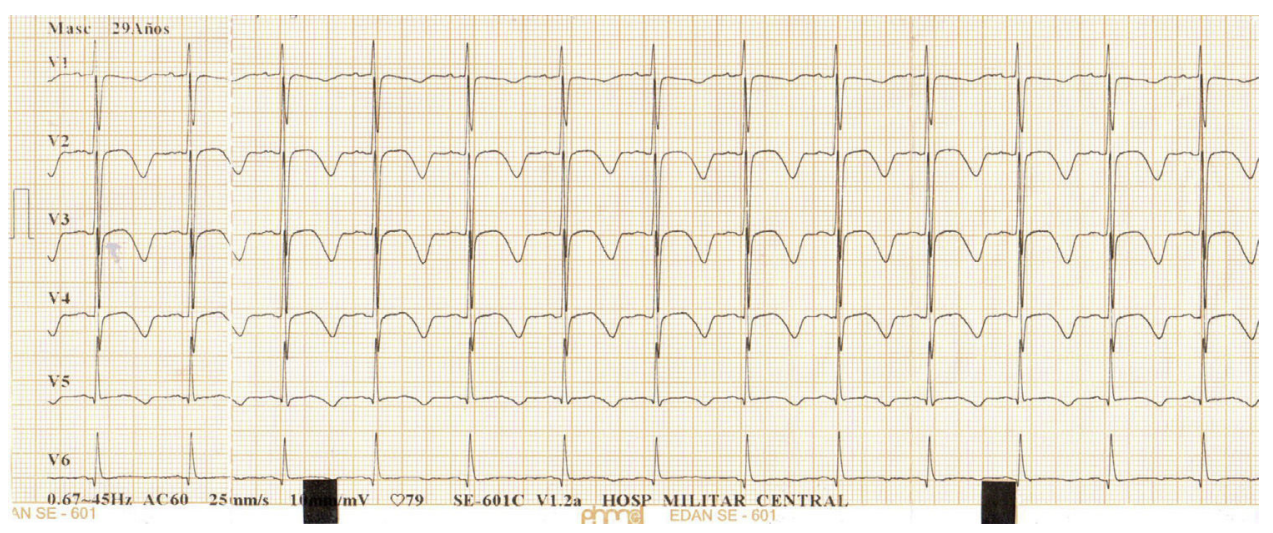

Figura 1

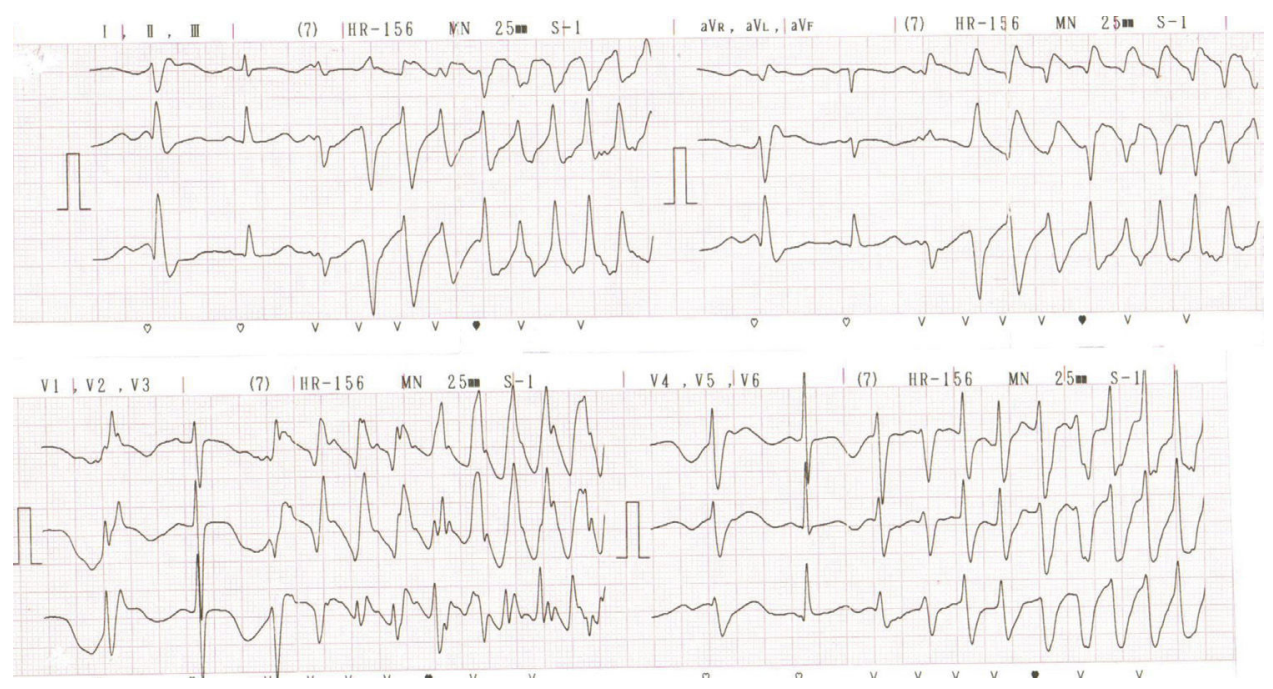

Figura 2

El SQTL adquirido y la torsade de pointes inducida por fármacos son producidos por una gran variedad de medicamentos de uso diario en la práctica clínica (consultar el portal www.qtdrugs.org $)^{4,5}$. Mientras otros canales de potasio tienen dos residuos de prolina, los cuales se inclinan en forma angulada hacia el poro central disminuyendo su lumen, el canal IKr (codificado por el gen KCNH2 - HERG) carece de estos aminoácidos en sus poros; adicionalmente, tiene dos residuos aromáticos (tirosina y fenilalanina) que forman enlaces con moléculas aromáticas presentes en los fármacos que bloquean el canal, lo cual facilita la interacción con los medicamentos con el canal $\mathrm{IKr}^{6}$. Los factores relacionados con la susceptibilidad individual al desarrollo del SQTL adquirido incluyen sexo femenino, hipocalcemia, hipocalemia, hipomagnesemia, bradicardia, hipertrofia ventricular izquierda, fibrilación auricular, poscardioversión eléctrica, falla cardiaca, alteraciones de las concentraciones séricas de los fármacos que lo predisponen y SQTL de penetrancia incompleta. Este último constituye los portadores asintomáticos de mutaciones que pueden manifestar arritmias malignas al recibir fármacos que desenmascaran la enfermedad ${ }^{1,4,7,8}$.

La toxicidad por antimoniales compromete los sistemas renal, nervioso, hematológico, respiratorio y cardiovascular.
Respecto a los mecanismos de toxicidad cardiaca se conoce que sus principales efectos se dan sobre la actividad eléctrica del corazón: prolongan el intervalo QT y alteran la morfología de la onda T del electrocardiograma, con el potencial desarrollo de arritmias fatales ${ }^{9}$. Sadeghian et al. ${ }^{10}$ realizaron un registro electrocardiográfico semanal en 131 pacientes con leishmaniasis cutánea hasta un mes después de finalizado el tratamiento con antimoniales. La prolongación del intervalo QT fue la anormalidad más frecuente, observada en el $19 \%$ de los pacientes (fue hasta de $520 \mathrm{~ms}$ en uno de los casos), seguido por bradicardia en el $10,6 \%$, depresión del segmento ST en el $6,1 \%$, elevación del segmento ST en el 3\%, inversión de la onda T en el 7,4\%, contracciones ventriculares prematuras en el $2,99 \%$ y bloqueo de rama izquierda en el $0,7 \%$ de los pacientes del estudio. Todos los cambios electrocardiográficos revirtieron con el retiro del medicamento.

Ribeiro et al. ${ }^{11}$, en 62 pacientes con leishmaniasis cutánea tratados con antimoniato de meglumina, encontraron un incremento de la duración del intervalo QTC que excedía los $40 \mathrm{~ms}$ en 12 pacientes; en 7 de ellos se desarrolló un QTc de por lo menos 500 ms. Shanehsaz et $\mathrm{al}^{12}$. evidenciaron la prolongación del intervalo QTc como la alteración electrocardiográfica más frecuente en 50 pacientes. 


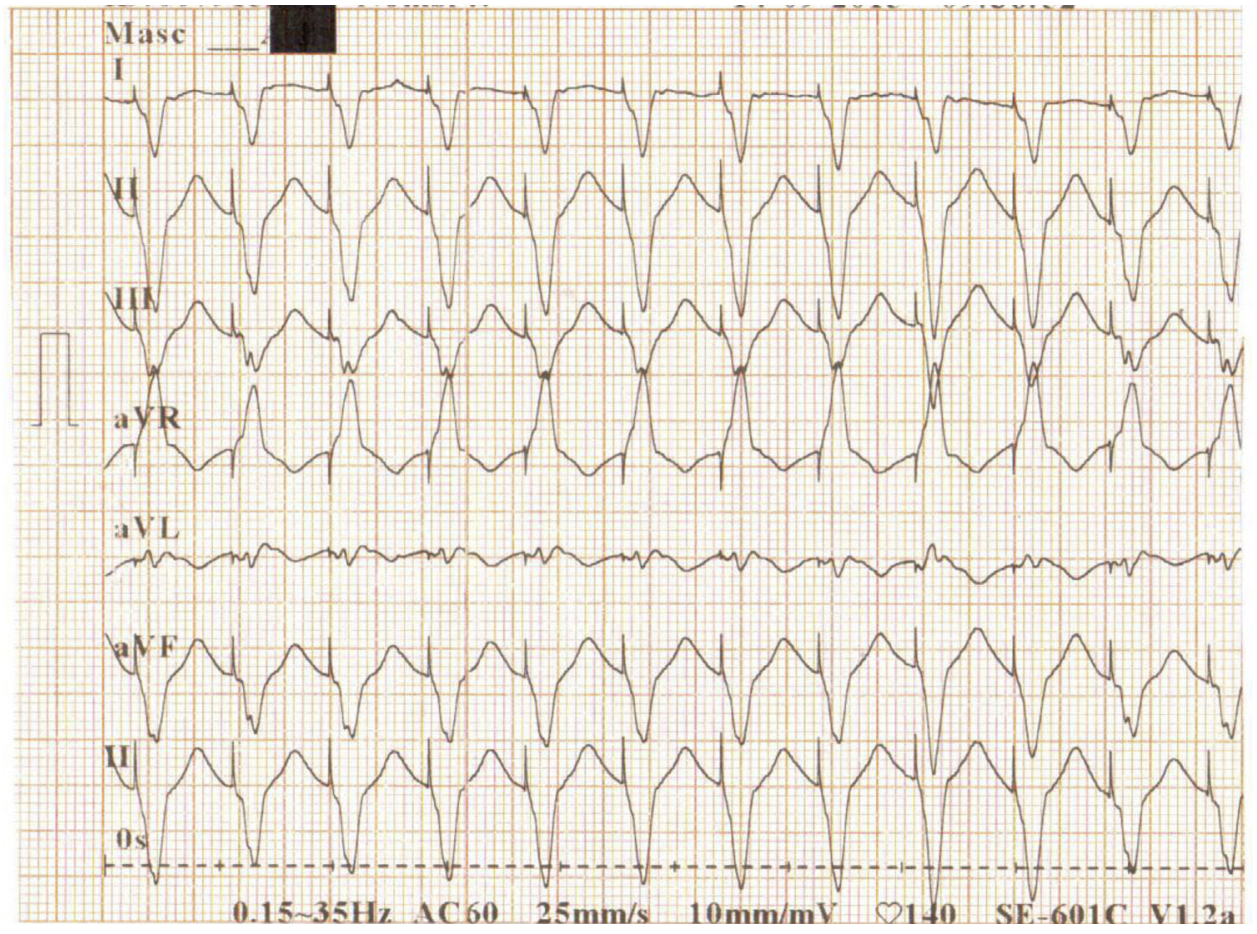

Figura 3

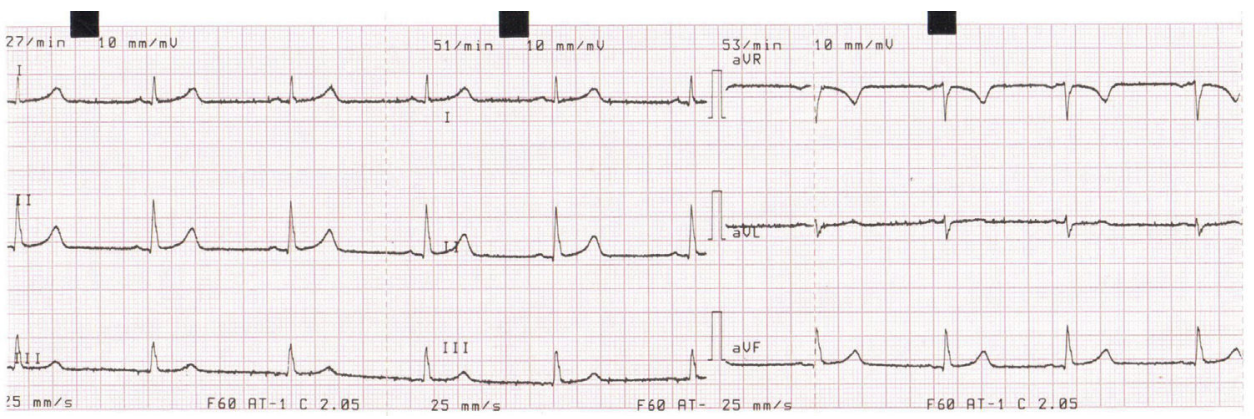

Figura 4

El intervalo QT puede prolongarse tanto por un incremento en la duración de la fase de despolarización (incremento en las corrientes de sodio o calcio) como por un retardo de la fase de repolarización (decremento en las corrientes de potasio) del potencial de acción ventricular. El mecanismo más común por el cual las drogas prolongan el intervalo QT es la inhibición directa de las canales de potasio que son responsables de las corrientes $\mathrm{IKr}^{13}$. El caso de los antimoniales parece ser diferente; Kuryshev et al. ${ }^{14}$ observaron que los antimoniales trivalentes reducen los canales de potasio hERG de una manera dependiente de concentración. Sin embargo, los antimoniales pentavalentes como el estibogluconato de sodio, no mostraron el mismo efecto sobre estas corrientes de potasio. En el mismo estudio, se evidenció que tanto los antimoniales trivalentes como los pentavalentes incrementan las corrientes cardiacas de calcio que regulan la fase de meseta del potencial de acción, produciendo un retardo en la repolarización cardiaca, lo cual puede explicar el riesgo de los pacientes tratados con antimoniales de desarrollar prolongación del intervalo QT y de arritmias potencialmente fatales como la torsade de pointes.

No existe un antídoto específico en el manejo de la toxicidad por antimoniales, por lo que el tratamiento de estos pacientes se basa en brindar medidas de soporte hasta la resolución del cuadro clínico. Kuryshev et al. ${ }^{14}$ observaron en su estudio que la acetil-cisteína contrarresta el efecto potenciador de los antimoniales pentavalentes sobre las corrientes de calcio de la fase de meseta del potencial de acción; sin embargo, no hay reportes en la literatura del uso de este medicamento en pacientes con toxicidad por glucantime. Un reporte de caso explora el papel potencial de los corticoides en la recuperación de los efectos secundarios de los antimoniales, observándose mejoría del QTc y de marcadores inflamatorios ${ }^{15}$. 


\section{Conclusiones}

Los antimoniales constituyen la terapia antiprotozoaria de primera línea en el tratamiento de pacientes con leishmaniasis cutánea; no obstante, esta puede asociarse a complicaciones potencialmente letales. El paciente del caso desarrolló toxicidad pancreática con disfunción multiorgánica y compromiso cardiaco manifestándose por un SQTL adquirido y múltiples episodios de taquicardia ventricular que incluían episodios de torsade de pointes.

Existe evidencia científica de la cardiotoxicidad de los antimoniales; sus efectos sobre los canales de calcio prolongan la duración del potencial de acción y por ende prolongan el intervalo QTc. Sin embargo, en este paciente se pudo constatar hipocalemia como factor que favorece el desarrollo de arritmias; igualmente es importante resaltar que durante la fase inicial de reanimación en urgencias se hizo uso de amiodora, medicación asociada a prolongación del intervalo QTc, que pudiera haber influido en la persistencia de los episodios de torsade de pointes.

\section{Conflicto de intereses}

Ninguno.

\section{Bibliografía}

1. Medeiros-domingo A, Iturralde-torres $P$, Ackerman J. Clínica y genética en el síndrome de QT largo. Rev Esp Cardiol. 2007;60:739-52.

2. Priori SG, Napolitano C, Schwartz PJ. Low penetrance in the long-QT syndrome: clinical impact. Circulation. 1999;12:978-90.
3. Priori SG. Inherited arrhythmogenic diseases: The complexity beyond monogenic disorders. Circ Res. 2004;94:140-5.

4. Viskin S, Justo D, Halkin A, Zeltser D. Long QT syndrome caused by noncardiac drugs. Prog Cardiovasc Dis. 2003;45:415-27.

5. Roden DM. Long-QT syndrome. N Engl J Med. 2008:169-76.

6. Abriel H, Schläpfer J, Keller DI, Gavillet B, Buclin T, Biollaz J, et al. Molecular and clinical determinants of drug-induced long QT syndrome: an iatrogenic channelopathy. Swiss Med Wkly. 2004;134(47-48):685-94.

7. Roden DM, Long QT. syndrome: reduced repolarization reserve and the genetic link. J Intern Med. 2006;259:59-69.

8. Kannankeril P, Roden DM, Darbar D. Drug-induced long QT syndrome. Pharmacol Rev. 2010;62:760-81.

9. Sundar S, Chakravarty J. Antimony Toxicity. Int J Environ Res Public Health. 2010;7:4267-77.

10. Sadeghian G, Ziaei H, Sadeghi M. Electrocardiographic changes in patients with cutaneous leishmaniasis treated with systemic glucantime. Ann Acad Med Singapore. 2008;37:916-8.

11. Ribeiro ALP, Drummond JB, Volpini AC, Andrade AC, Passos VMA. Electrocardiographic changes during low-dose, short-term therapy of cutaneous leishmaniasis with the pentavalent antimonial meglumine. Brazilian journal of medical and biological research. 1999;32:297-301.

12. Shanehsaz SM, Ishkhanian S. Electrocardiographic and biochemical adverse effects of meglumine antimoniate (MA) during treatment of Syrian cutaneous leishmaniasis patients. J Pakistan Assoc Dermatologists. 2013;23:412-7.

13. Bjerregaard P, Gussak I. Electrical diseases of the heart. 2013:2.

14. Kuryshev YA, Wang L, Wible BA, Wan X, Ficker E. Antimony-based antileishmanial compounds prolong the cardiac action potential by an increase in cardiac calcium currents. Mol Pharmacol. 2006;69:1216-25.

15. Brostoff JM, Lockwood DN. Glucocorticoids as a novel approach to the treatment of disabling side effects of sodium stiboglconate. J Clin Pharm Ther. 2012;37:122-213. 